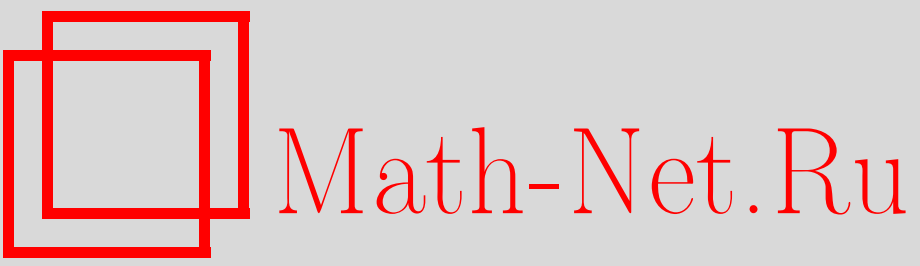

М. Бордаг, В. В. Скалозуб, Суммирование всех лепестковых диаграмм в $O(N)$ модели вблизи температуры фазового перехода, ТМФ, 2002, том 131, номер 1, 414

DOI: https://doi.org/10.4213/tmf1943

Использование Общероссийского математического портала Math-Net.Ru подразумевает, что вы прочитали и согласны с пользовательским соглашением

http://www.mathnet.ru/rus/agreement

Параметры загрузки:

IP : 52.87 .193 .239

26 апреля 2023 г., 14:35:34 
Том 131, № 1

апрель, 2002

(C) 2002 г.

М. Бордаг ${ }^{*}$ В. Скалозуб ${ }^{\dagger}$

\section{СУММИРОВАНИЕ ВСЕХ ЛЕПЕСТКОВЫХ ДИАГРАММ В $O(N)$-МОДЕЛИ ВБЛИЗИ ТЕМПЕРАТУРЫ ФАЗОВОГО ПЕРЕХОДА}

Исследован температурный фазовый переход в теории $N$-компонентного скалярного поля. В приближении суммирования всех диаграмм лепесткового типа получен слабый фазовый переход первого рода. При $N \rightarrow \infty$ он становится фазовым переходом второго рода. Проведено сравнение с другими подходами.

\section{1. ВВЕДЕНИЕ}

Исследования фазового перехода в $N$-компонентной $\phi^{4}$-теории при конечной температуре проводились в течение многих лет различными методами. Интерес к этой теории обусловлен, в частности, тем, что для $N=4$ она описывает скалярный сектор стандартной электрослабой модели, а для некоторых больших $N$ соответствует ее различным расширениям. Для отрицательного квадрата голой массы в теории возникает спонтанное нарушение $O(N)$ симметрии до $O(N-1)$. При достаточно высокой температуре исходная симметрия восстанавливается [1], [2]. Тип фазового перехода, определенный в пертурбативных (см., например, [3]-[5]) и в непертурбативных (решеточные симуляции, усредненное действие) [6]-[10] методах разложения при больших $N$, различен. В первом случае обнаружен фазовый переход первого рода, тогда как во втором - второго рода. По обшему мнению, это несоответствие обусловлено потерей сходимости ряда теории возмушений вблизи критической температуры. В работах [11], [4], [12], используюших пертурбативные методы, просуммированы так называемые лепестковые диаграммы. В работах [13], [14] с помощью метода вспомогательной массы обнаружено, что фазовый переход как в $O(1)$-, так и в $O(N)$-моделях - второго рода. Ввиду важности этих результатов, мы обсудим их ниже более подробно. Здесь же только обратим внимание на то,

\footnotetext{
${ }^{*}$ University of Leipzig, Institute for Theoretical Physics, Leipzig, Germany. E-mail: Michael.Bordag@itp.uni-leipzig.de

$\dagger$ Днепропетровский государственный университет, Днепропетровск, Украина. E-mail: Skalozub@ff.dsu.dp.ua
} 
что вычисленный в статьях [13], [14] эффективный потенциал имеет в минимуме мнимую часть. Это означает несостоятельность примененной вычислительной процедуры.

В нашей предыдущей статье [15] проведен подробный анализ фазового перехода в $O(1)$-модели в приближении суммирования всех лепестковых диаграмм и класса диаграмм более высокого порядка. Для усовершенствования вычислений был развит новый метод, совмешаюший второе преобразование Лежандра с решением уравнений шели в экстремумах функционала свободной энергии. Это позволяет устранить конденсат, возникаюший в результате спонтанного нарушения симметрии, как переменную из уравнений шели и значительно упрощает расчеты. В указанном приближении мы получили фазовый переход первого рода и предполагавшееся исчезновение эффективного параметра разложения вблизи температуры фазового перехода. Было показано также, что минимум эффективного потенциала в сделанном приближении является действительным, как это и следует из соображений самосогласованности метода вычислений.

В данной статье мы применяем метод работы [15] к $O(N)$-модели. Показано, что разрешение уравнений шели в приближении суммирования всех лепестковых диаграмм в минимуме свободной энергии приводит к фазовому переходу первого рода для любого конечного $N$. Он становится переходом второго рода в пределе $N \rightarrow \infty$. Наблюдаемый фазовый переход весьма близок к фазовому переходу второго рода и отличается от него в следуюшем после основного порядке по $1 / N$ для конечных, но больших $N$.

План дальнейшего изложения следуюший. В разделе 2 приведены необходимые сведения о преобразовании Лежандра второго рода и уравнениях Швингера-Дайсона. В разделе 3 решены уравнения шели в приближении суммирования всех лепестковых диаграмм для произвольного $N$. Раздел 4 посвящен обсуждению результатов.

\section{2. ОБЩИЕ СООБРАЖЕНИЯ}

Рассмотрим теорию скалярного $N$-компонентного поля размерности $3+1$ в евклидовом пространстве. Действие имеет вид

$$
S[\phi]=\int d x\left(\frac{1}{2} \phi(x) K \phi(x)-\frac{\lambda}{4 N}(\phi(x))^{4}\right)
$$

где $K=\square-m^{2}$ - ядро свободного действия. Знак выбран таким образом, чтобы вакуумные функции Грина определялись функциональным представлением

$$
Z \equiv e^{W}=\int D \phi e^{S}
$$

где $W$ - функционал для связных функций Грина. Он выражается через свободную энергию $F$ с помошью соотношения:

$$
W=-F T,
$$


где $T$ - температура. Мы рассматриваем теорию при конечной температуре в формализме мнимого времени, так что интегрирование по петлям дается формулой

$$
\operatorname{Tr}_{p}=T \sum_{l=-\infty}^{\infty} \int \frac{d^{3} \vec{p}}{(2 \pi)^{3}}
$$

с импульсом $p=(2 \pi T l, \vec{p}) \quad(l \in Z)$. Символом $\phi$ в формуле (1) обозначена совокупность $N$ скалярных полей, $\phi=\left\{\phi_{1}, \phi_{2}, \ldots, \phi_{N}\right\}$.

В действии (1) предполагается следуюшее суммирование по внутренним индексам:

$$
\phi^{2}=\sum_{a=1}^{N} \phi_{a}^{2}, \quad \phi^{4}=\left(\sum_{a=1}^{N} \phi_{a}^{2}\right)^{2} .
$$

Перенормировка производится при нулевой температуре. Второе преобразование Лежандра приводит к представлению [16]

$$
W=S[0]+\frac{1}{2} \operatorname{Tr} \ln \beta-\frac{1}{2} \operatorname{Tr} \Delta^{-1} \beta+W_{2}[\beta]
$$

для связных функций Грина, где пропагатор $\beta$ удовлетворяет уравнению ШвингераДайсона (ШД)

$$
\beta^{-1}(p)=\Delta^{-1}-\Sigma[\beta](p)
$$

и $\Delta=-K^{-1}-$ свободный пропагатор. Здесь $\Sigma[\beta](p)-$ функционал всех графов собственной энергии без пропагаторных вставок,

$$
\Sigma[\beta](p)=2 \frac{\delta W_{2}}{\delta \beta(p)},
$$

где берется функциональная производная (см. подробнее [16]), $W_{2}[\beta]$ - сумма всех двухчастично неприводимых графов, полученных из связных функций Грина,

$$
W_{2}[\beta]=\sum W[\beta]
$$

с функцией $\beta(p)$ на линиях.

Приведенные формулы записаны в обобшенных обозначениях. Ниже мы выпишем их явно для $O(N)$-модели. Но прежде чем это сделать, введем спонтанное нарушение симметрии путем обрашения знака массового члена $m^{2} \rightarrow-m^{2}$ в свободном пропагаторе $\Delta$. Тогда на древесном уровне минимум энергии достигается путем сдвига полей $\phi_{1} \rightarrow \eta+v$, где $v$ - конденсат. Затем заменим обозначения $\left\{\phi_{1}, \phi_{2}, \ldots, \phi_{N}\right\} \rightarrow$ $\left\{\eta+v, \phi_{1}, \phi_{2}, \ldots, \phi_{N-1}\right\}$, где $\eta$ - поле Хиггса и $\left\{\phi_{1}, \phi_{2}, \ldots, \phi_{N-1}\right\}$ - голдстоуновские поля, симметричные относительно $O(N-1)$-преобразований. 
После этих операций действие принимает вид

$$
\begin{aligned}
S[\eta, \phi] & =\int d x\left[\frac{m^{2}}{2} v^{2}-\frac{\lambda}{4 N} v^{4}+\eta\left(m^{2}-\frac{\lambda}{N} v^{2}\right) v+\right. \\
& \left.+\frac{1}{2} \eta\left(\square-\mu_{\eta}^{2}\right) \eta+\frac{1}{2} \phi\left(\square-\mu_{\phi}^{2}\right) \phi-\frac{\lambda}{4 N}\left(\eta^{4}+4 \eta^{3} v+2\left(\eta^{2}+2 \eta v\right) \phi^{2}+\phi^{4}\right)\right]
\end{aligned}
$$

где

$$
\mu_{\eta}=-m^{2}+3 \frac{\lambda}{N} v^{2}, \quad \mu_{\phi}=-m^{2}+\frac{\lambda}{N} v^{2}
$$

и по-прежнему предполагается суммирование по внутренним индексам. В импульсном представлении соответствуюшие свободные пропагаторы имеют вид

$$
\Delta_{\eta}=p^{2}+\mu_{\eta}^{2}, \quad \Delta_{\phi}=p^{2}+\mu_{\phi}^{2}
$$

Пропагатор голдстоуновских бозонов диагонален $\left(\Delta_{\phi}\right)_{a b}=\delta_{a b} \Delta_{\phi}$. В этих обозначениях представление (5) для связных вакуумных функций Грина принимает вид

$$
\begin{aligned}
W= & S[0]+\frac{1}{2} \operatorname{Tr} \ln \beta_{\eta}+\frac{N-1}{2} \operatorname{Tr} \ln \beta_{\phi}- \\
& -\frac{1}{2} \operatorname{Tr} \Delta_{\eta}^{-1} \beta_{\eta}-\frac{N-1}{2} \operatorname{Tr} \Delta_{\phi}^{-1} \beta_{\phi}+W_{2}\left[\beta_{\eta}, \beta_{\phi}\right],
\end{aligned}
$$

где $W_{2}\left[\beta_{\eta}, \beta_{\phi}\right]$ - сумма по всем двухчастично неприводимым графам с пропагаторами $\beta_{\eta}$ и $\beta_{\phi}$ на линиях, соответствующим полям $\eta$ и $\phi$. В модели $O(N)$ в симметричной фазе уравнение ШД образует набор $N$ уравнений, эквивалентных одному. В случае нарушенной симметрии остается два уравнения,

$$
\begin{aligned}
& \beta_{\eta}^{-1}(p)=\Delta_{\eta}^{-1}-\Sigma_{\eta}\left[\beta_{\eta}, \beta_{\phi}\right](p), \\
& \beta_{\phi}^{-1}(p)=\Delta_{\phi}^{-1}-\Sigma_{\phi}\left[\beta_{\eta}, \beta_{\phi}\right](p),
\end{aligned}
$$

где аналогично (7) функционал собственной энергии дается выражением

$$
\begin{aligned}
& \Sigma_{\eta}\left[\beta_{\eta}, \beta_{\phi}\right](p)=2 \frac{\delta W_{2}\left[\beta_{\eta}, \beta_{\phi}\right]}{\delta \beta_{\eta}(p)} \\
& \Sigma_{\phi}\left[\beta_{\eta}, \beta_{\phi}\right](p)=\frac{2}{N-1} \frac{\delta W_{2}\left[\beta_{\eta}, \beta_{\phi}\right]}{\delta \phi_{\eta}(p)} .
\end{aligned}
$$

Здесь использовано соотношение

$$
\left(\Sigma_{\phi}\right)_{a b}=\delta_{a b} \Sigma_{\phi}=2 \frac{\delta W_{2}}{\delta\left(\beta_{\phi}\right)_{a b}}=\delta_{a b} \frac{2}{N-1} \frac{\delta W_{2}}{\delta \beta_{\phi}} .
$$

При достаточно высокой температуре симметрия восстанавливается. Это означает, что величина коденсата $v=0$ и массы хиггсовских и голдстоуновских частиц равны, $M_{\eta}=$ 
$M_{\phi}$. Тогда все уравнения ШД сводятся к одному (6). При низкой температуре симметрия нарушена, $v \neq 0$, массы различны и нужно рассматривать два уравнения (12). Свободная энергия становится, таким образом, функцией конденсата и имеет при $v>0$ минимум, который возникает уже на древесном уровне. Функционал может иметь и другие экстремумы. В этом случае, ввиду непрерывности свободной энергии как функции $v$, дополнительный экстремум для $v>0$ должен быть максимумом, означающим наличие фазового перехода первого рода.

Далее, следуя работе [15], рассмотрим свободную энергию в ее экстремумах. Вычислим первую производную $W$ из уравнения (11)

$$
\frac{d}{d v^{2}} W\left[\beta_{\eta}, \beta_{\phi}\right]=\frac{m^{2}}{2}-\frac{\lambda}{2 N} v^{2}-\frac{3 \lambda}{2 N} \operatorname{Tr} \beta_{\eta}-\frac{\lambda(N-1)}{2 N} \operatorname{Tr} \beta_{\phi} .
$$

В этом выражении присутствуют, вообше говоря, вклады, пропорциональные $\partial \beta_{\eta} / \partial v^{2}$ и $\partial \beta_{\phi} / \partial v^{2}$. Однако они исчезают при учете уравнений ШД (12). Здесь мы также приняли во внимание, что в приближении суммирования всех лепестковых диаграмм интересуюший нас член $W_{2}\left[\beta_{\eta}, \beta_{\phi}\right]$ не зависит от $v^{2}$ (см. ниже формулу (17)). Требуя теперь обрашения в нуль производной, определяемой уравнением (14),

$$
\frac{d W\left[\beta_{\eta}, \beta_{\phi}\right]}{d v^{2}}=0
$$

получаем

$$
\frac{\lambda}{N} v^{2}=m^{2}-3 \frac{\lambda}{N} \operatorname{Tr} \beta_{\eta}-\frac{\lambda(N-1)}{N} \operatorname{Tr} \beta_{\phi} .
$$

Уравнение (16) должно рассматриваться вместе с уравнениями ШД (12).

Обратимся теперь к приближению суммирования всех лепесковых диаграмм. Такое приближение обеспечивается сохранением в функционале $W_{2}$ графов только с одной вершиной

$$
\begin{aligned}
W_{2}^{\mathrm{SD}} & =\frac{1}{8}+\frac{1}{4}+\frac{1}{4} \cdot\left(\Sigma_{\eta}^{(0)}\right)^{2}-\frac{\lambda}{2} \frac{N-1}{N} \Sigma_{\eta}^{(0)} \Sigma_{\phi}^{(0)}-\frac{\lambda}{2} \frac{N^{2}-1}{N}\left(\Sigma_{\phi}^{(0)}\right)^{2} . \\
& =-\frac{3 \lambda}{4 N}(
\end{aligned}
$$

Здесь мы ввели обозначения $\Sigma_{\eta}^{(0)}=\operatorname{Tr} \beta_{\eta}$ и $\Sigma_{\phi}^{(0)}=\operatorname{Tr} \beta_{\phi}$ для графов, состоящих из одной линии, замкнутой через одну вершину.

Вернемся теперь к уравнениям ШД. В данном приближении графы собственной энергии в правой части уравнений (12) не зависят от импульса $p$. Следовательно, подстановка

$$
\beta_{\eta}^{-1}=p^{2}+M_{\eta}^{2}, \quad \beta_{\phi}^{-1}=p^{2}+M_{\phi}^{2}
$$

для пропагаторов становится точной и уравнения ШД сводятся к шелевым уравнениям для соответствуюших масс. 
В восстановленной фазе конденсат $v=0$ и массы $M_{\eta}=M_{\phi} \equiv M_{r}$, где

$$
M_{r}^{2}=-m^{2}+\lambda \frac{N+2}{N} \Sigma^{(0)}
$$

В фазе с нарушенной симметрией конденсат в экстремуме свободной энергии дается уравнением (16) и уравнения (12) можно переписать в виде

$$
\begin{aligned}
& M_{\eta}^{2}=-m^{2}+\frac{3 \lambda}{N} v^{2}+\frac{3 \lambda}{N} \Sigma_{\eta}^{(0)}+\lambda \frac{N-1}{N} \Sigma_{\phi}^{(0)}, \\
& M_{\phi}^{2}=-m^{2}+\frac{\lambda}{N} v^{2}+\frac{\lambda}{N} \Sigma_{\eta}^{(0)}+\lambda \frac{N+1}{N} \Sigma_{\phi}^{(0)} .
\end{aligned}
$$

Уравнение (16) следует из условия экстремума, представленного в виде

$$
\frac{\lambda v^{2}}{N}=m^{2}-\frac{3 \lambda}{N} \Sigma_{\eta}^{(0)}-\lambda \frac{N-1}{N} \Sigma_{\phi}^{(0)} .
$$

Используем соотношение (22) для преобразования первого уравнения шели (20),

$$
M_{\eta}^{2}=2\left(m^{2}-\frac{3 \lambda}{N} \Sigma_{\eta}^{(0)}-\lambda \frac{N-1}{N} \Sigma_{\phi}^{(0)}\right) .
$$

Уравнение (22) можно записать в виде

$$
\frac{\lambda}{N} v^{2}=\frac{M_{\eta}^{2}}{2}
$$

В этом случае уравнение (21) записьвается как

$$
M_{\phi}^{2}=\frac{2 \lambda}{N}\left(\Sigma_{\phi}^{(0)}-\Sigma_{\eta}^{(0)}\right)
$$

Таким образом, все необходимые обшие формулы получены, и мы можем приступить к решению этих уравнений.

\section{3. РЕШЕНИЕ УРАВНЕНИЙ ШЕЛИ}

Решение шелевых уравнений (19), (23) и (25) может быть исследовано численно для некоторых $\lambda$ и $T$. Нас интересует малая затравочная константа связи $\lambda$. Вблизи фазового перехода температура велика, $T \sim 1 / \sqrt{\lambda}$. Отсюда получаем известное высокотемпературное разложение для функции $\Sigma^{(0)}$,

$$
\Sigma^{(0)}=\frac{T^{2}}{12}-\frac{M T}{4 \pi}+\cdots
$$

где нужно подставить соответствующие массы $M_{r}, M_{\eta}$ или $M_{\phi}$. В уравнении (26) точки обозначают вклады, подавленные более высокими степенями $M / T$. Они давали бы 
только малую поправку к полученным ниже результатам. Заметим, что сохраненные в (26) члены не содержат ультрафиолетовых расходимостей и соответствующий произвол конечной перенормировки присутствует только в первых опушенных членах.

В этом приближении шелевые уравнения дают для фазы с восстановленной симметрией

$$
M_{r}^{2}=-m^{2}+\frac{\lambda(N+2) T^{2}}{12 N}-2 M_{r} \frac{\lambda(N+2) T}{8 \pi N}
$$

В фазе с нарушенной симметрией

$$
\begin{aligned}
& M_{\eta}^{2}=2 m^{2}-\frac{\lambda(N+2) T^{2}}{6 N}+2 M_{\eta} \frac{3 \lambda T}{4 \pi N}+M_{\phi} \frac{\lambda(N-1) T}{2 \pi N} \\
& M_{\phi}^{2}=\frac{\lambda T}{2 \pi N}\left(M_{\eta}-M_{\phi}\right) .
\end{aligned}
$$

В восстановленной фазе уравнение (27) решается просто,

$$
M_{r}=-\frac{\lambda(N+2) T}{8 \pi N}+\sqrt{\left(\frac{\lambda(N+2) T}{8 \pi N}\right)^{2}-m^{2}+\frac{\lambda(N+2) T^{2}}{12 N}}
$$

Видно, что уравнение (27) имеет единственное решение для положительной массы при $T>T_{-}$, где

$$
T_{-}=\sqrt{\frac{12 N}{\lambda(N+2)}} m
$$

- температура нижней спинодали.



Рис. 1 
В нарушенной фазе два уравнения щели (28) образуют систему алгебраических уравнений. Второе уравнение (28) имеет единственное решение для всех $T$ относительно $M_{\phi}$,

$$
M_{\phi}=-\frac{\lambda T}{4 \pi N}+\sqrt{\left(\frac{\lambda T}{4 \pi N}\right)^{2}+\frac{\lambda T}{2 \pi N} M_{\eta}}
$$

Заметим, что при $M_{\eta}=0$ частное значение $M_{\phi}=0$ не зависит от $T$. Подставляя $M_{\phi}$ из (31) в первое уравнение (28), имеем

$$
M_{\eta}^{2}=2 m^{2}-\frac{\lambda(N+2) T^{2}}{6 N}+2 M_{\eta} \frac{3 \lambda T}{4 \pi N}+\frac{\lambda(N-1) T}{2 \pi N} M_{\phi}\left(M_{\eta}\right) .
$$

Это уравнение имеет явное решение, которое весьма громоздко и здесь не приводится. Оно легко изображается графически и показано на рис. 1 , где изображены массы $M_{\eta}$, $M_{\phi}$ и $M_{r}$ как функции температуры $T$ для $\lambda=1, N=2$. Верхние части кривых для $M_{\eta}$ и $M_{\phi}$ дают соответствующие массы в точке минимума свободной энергии, нижние части - массы в точке максимума.. Мы выбрали $\lambda=1$, чтобы сделать более различимыми подробности вблизи точки фазового перехода. Можно получить основные свойства этого решения. Для малого $T$ сушествует одно решение. При $T=T_{-}$появляется второе решение, и при некоторой температуре $T_{+}$оба решения сливаются и исчезают. Следовательно, значение $T_{+}$интерпретируется как температура верхней спинодали. Таким образом, мы наблюдаем фазовый переход первого рода. Поскольку у нас есть алгебраическое решение уравнений шели, можно выписать алгебраическое выражение для $T_{+}$. Оно также слишком сложно и здесь не приводится. Однако для малых $\lambda$ значение $T_{+}$ можно выразить как

$$
\frac{T_{+}}{T_{-}}=1+t_{N} \lambda+O\left(\lambda^{2}\right),
$$

где числа $t_{N}$ алгебраически зависят от $N$. Некоторые значения этих чисел даны в таблище.

\begin{tabular}{|c|c|c|c|c|c|c|}
\hline$N$ & 0 & 1 & 2 & 3 & 4 & 5 \\
\hline$t_{N}$ & 0.0569932 & 0.029511 & 0.0203949 & 0.0158314 & 0.0130797 & 0.0112318 \\
\hline
\end{tabular}

Для $N=1$ мы получаем еше раз $\left(\right.$ с $\left.t_{1}=9 /\left(16 \pi^{2}\right)\right)$ известный результат для однокомпонентного случая [15]. Можно показать, что $t_{N} \rightarrow 0$ при $N \rightarrow \infty$.

Теперь, решив уравнение шели, рассмотрим функционал $W$. Пусть $W_{b}$ - его значение в точке минимума (нарушенная фаза). При этом массы определены уравнениями (28), а конденсат - уравнением (22). Пусть также $W_{r}=W\left(0, M_{r}, M_{r}\right)$ - значение функционала $W$ в восстановленной фазе. Соответствующие аналитические выражения снова слишком громоздки и приводить их здесь излишне. Поэтому мы ограничимся некоторыми численными значениями. Например, для $\lambda=1$ и $N=2$ имеем $T_{-}=2.44949$ 


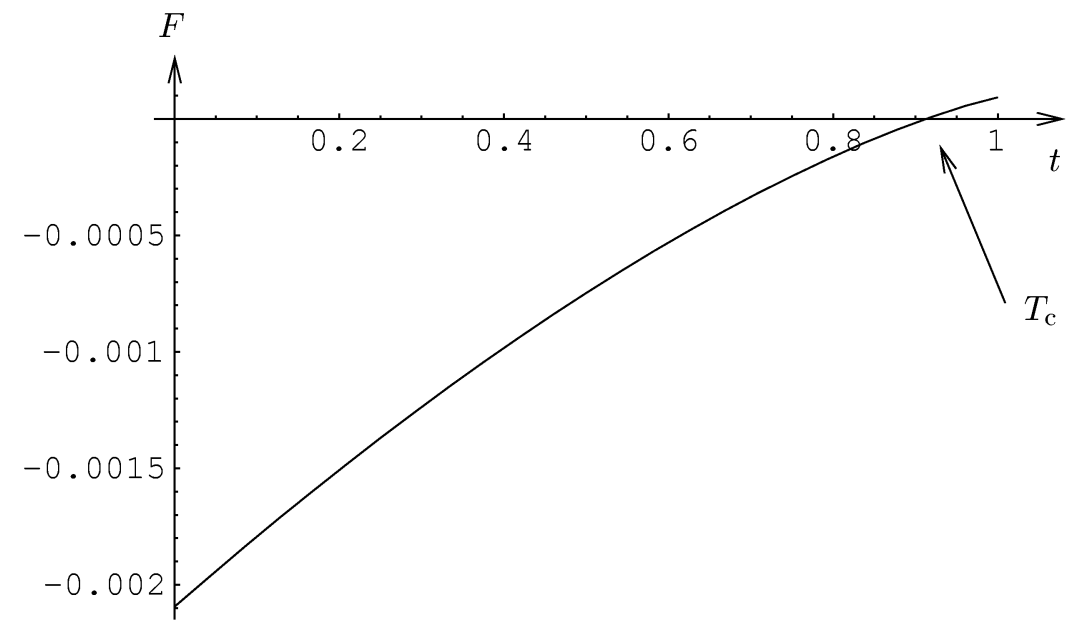

Рис. 2

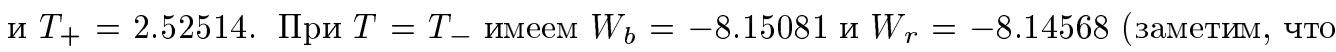
свободная энергия отличается от $W$ знаком, см. (3)). При $T=T_{+}$соответствующие значения $W_{b}=-8.15081$ и $W_{r}=-9.1671$. На рис. 2 изображена свободная энергия $F=-\Delta W / T$ как функция температурного параметра $t\left(T=T_{-}+t\left(T_{+}-T_{-}\right)\right)$в области фазового перехода, т.е. для $T_{-} \leqslant T \leqslant T_{+}$. Из условия $\Delta W \equiv W_{b}-W_{r}=0$ находим температуру фазового перехода $T_{\mathrm{c}}=2.5198$.

\section{4. АНАЛИЗ РЕЗУЛЬТАТОВ. ЗАКЛЮЧИТЕЛЬНЫЕ ВЫВОДЫ}

Мы исследовали фазовый переход в модели $O(N)$ пертурбативными методами. Были использованы преобразование Лежандра второго рода и метод анализа уравнений шели в экстремуме свободной энергии. Последний метод значительно упрошает выражения (так, например, не возникает однопетлевых графов с одной внешней линией "головастик") и позволяет получить результат в более явном виде. В приближении суммирования всех лепестковых диаграмм для малой константы связи $\lambda$ получается фазовый переход первого рода, что обобшает соответствуюшие результаты для однокомпонентного случая [15]. Массы хиггсовских и голдстоуновских частиц в нарушенной фазе и масса поля в восстановленной фазе показаны на рис. 1 , соответствуюшая свободная энергия приведена на рис. 2. Стоит отметить, что характер фазового перехода как перехода первого рода выражен довольно слабо. Как видно из рис. 2 , изменение свободной энергии меж ду $T_{\mathrm{c}}$ и $T_{+}$численно мало по сравнению с массовой шкалой (масса на рис. 2 принята за единицу). Кроме того, различие между двумя спинодальными температурами (33) имеет порядок $\lambda$. Таким образом, переход второго рода проявляется только в следуюшем порядке по $\lambda$ и $1 / N$. Точно таким же путем, как в однокомпонентном случае, можно показать, что эффективный параметр разложения для более высоких петлевых вкладов имеет первый порядок. 
Полученные результаты оставляют нерешенной сложную проблему, состояшую в том, что непертурбативные подходы (решеточные расчеты, уравнения потока и т.д.), равно как и некоторые пертурбативные расчеты [17], предсказывают для фазового перехода характер второго рода. Рассмотрим этот вопрос подробнее.

В статье [17] использован метод ренормгруппы при конечной температуре. Результаты, полученные в этом подходе, трудно сравнить с найденными в случае стандартной перенормировки при нулевой температуре. Причина состоит в том, что при конечной температуре перенормировки эффективно пересуммируют некоторые ряды диаграмм, остающиеся не вполне корректно определенными.

В статьях [13], [14] был разработан метод непертурбативного расчета эффективного потенциала при конечной температуре - метод вспомогательного поля. Фазовый переход второго рода наблюдался в однокомпонентной и $N$-компонентных моделях. Как упоминалось во введении, этот подход представляется нам не вполне самосогласованным, так как у эффективного потенциала в его минимуме возникает мнимая часть. Этот важный пункт является критическим для вычислительных процедур в целом. Действительно, минимум эффективного потенциала описывает физические вакуумные состояния системы. Мнимая часть сигнализирует либо о ложном вакууме, либо о несостоятельности использованной расчетной процедуры. Этот хорошо известный основополагающий момент восходит к работе [2], где указана необходимость пересуммирования для сохранения эффективного потенциала действительным. В нашем методе расчета это требование удовлетворяется автоматически, когда все лепестковые диаграммы суммируются в экстремумах свободной энергии. Стоит также упомянуть работу [18], где приведены аргументы в пользу невозможности фазового перехода второго рода в инфракрасно-стабильных теориях, хотя выдвигались также и противоположные утверждения [19]

Обычно принято считать, что суммирование других классов диаграмм (по отношению к лепестковым диаграммам) будет придавать фазовому переходу характер второго рода. Специфический вид диаграмм, ответственных за это важное изменение, также еще не определен. Более того, как было установлено в статье [15], некоторые классы диаграмм сверх лепестковых диаграмм могут усиливать характер первого рода в фазовом переходе. В частности этим свойством обладают диаграммы типа "цепочки пузырей". В случае потери малого параметра разложения вблизи температуры фазового перехода, обнаруженной в приближении "всех лепестков", интересен не только поиск нового класса диаграмм для пересуммирования, но и более подробный анализ самого класса лепестковых диаграмм.

\section{Список литературы}

[1] J. Zinn-Justin. Quantum Field Theory and Critical Phenomena. Oxford: Clarendon Press, 1996.

[2] L. Dolan, R. Jackiw. Phys. Rev. D. 1974. V. 9. P. 3320-3341.

[3] P. Arnold, O. Espinosa. Phys. Rev. D. 1993. V. 47. P. 3546-3579.

[4] M.E. Carrington. Phys. Rev. D. 1992. V. 45. P. 2933-2944.

[5] J. R. Espinosa, M. Quiros, F. Zwirner. Phys. Lett. B. 1992. V. 291. P. 115-124. 
[6] N. Tetradis, C. Wetterich. Nucl. Phys. B. 1993. V. 398. P. 659-696.

[7] M. Reuter, N. Tetradis, C. Wetterich. Nucl. Phys. B. 1993. V. 401. P. 567-590.

[8] J. Adams et al. Mod. Phys. Lett. A. 1995. V. 10. P. 2367-2380.

[9] I. Montvay, G. Muenster. Quantum Fields on a Lattice (Cambridge monographs on Mathematical Physics). Cambridge: Cambridge Univ. Press, 1994.

[10] Z. Fodor, J. Hein, K. Jansen, A. Jaster, I. Montvay. Nucl. Phys. B. 1995. V. 439. P. 147-186.

[11] K. Takahashi. Z. Phys. C. 1985. V. 26. P. 601-613.

[12] P. Arnold. Phys. Rev. D. 1992. V. 46. P. 2628-2635.

[13] K. Ogure, J. Sato. Phys. Rev. D. 1998. V. 57. P. 7460-7466.

[14] K. Ogure, J. Sato. Phys. Rev. D. 1998. V. 58. P. 085010.

[15] M. Bordag, V. Skalozub. J. Phys. A. 2001. V. 34. P. 461-471.

[16] J. M. Cornwall, R. Jackiw, E. Tomboulis. Phys. Rev. D. 1974. V. 8. P. 2428-2445.

[17] P. Elmfors. Z. Phys. C. 1992. V. 56. P. 601-608.

[18] S. Mallik, Krishnendu Mukherjee. Absence of a second order phase transition in $\lambda \phi^{4}$ theory. hep-th/9607087.

[19] P. Ginsparg. Nucl. Phys. B. 1980. V. 170(FS1). P. 388-408. 